\title{
$J^{P C}$-Exotic Mesons from the Bethe-Salpeter Equation
}

\author{
C. J. Burden \\ Department of Theoretical Physics, Research School of Physical Sciences and Engineering, \\ Australian National University, Canberra, ACT 0200, Australia \\ M. A. Pichowsky \\ Center for Nuclear Research, Department of Physics, \\ Kent State University, Kent, OH 44242, U.S.A.
}

\begin{abstract}
Masses for vector- $1^{--}$, axialvector- $1^{+-}, 1^{++}$, and exotic-vector $1^{-+}$mesons are calculated within a quantum field theoretic framework based on the Dyson-Schwinger equations using a model form of the quark-antiquark interaction that is separable. The model provides an excellent description of $\pi$ and $K$-meson observables and the flavor-octet ground-state meson spectrum. With no adjustments to model parameters, a numerical solution of the Bethe-Salpeter equation yields two exotic- $1^{-+}$ mesons with masses of 1439 and $1498 \mathrm{MeV}$.
\end{abstract}

\section{INTRODUCTION}

The $\pi_{1}(1400)$ meson, which was until recently referred to as $\hat{\rho}(1405)$, may be the first direct evidence of a non$q \bar{q}$ meson. With an estimated mass of $1376 \pm 17 \mathrm{MeV}$ and width of $300 \pm 40 \mathrm{MeV}$ [1], this resonance is distinguished by the exotic quantum numbers $J^{P C}=1^{-+}$. Evidence of this resonance was observed in $\pi p$ scattering by the E852 Collaboration [2] at Brookhaven and in $\bar{p} d$ annihilation by the Crystal Barrel Collaboration [3] at CERN. Difficulties encountered in extracting information about this resonance have raised some doubts as to its existence. However, more recently, a second observed exotic- $1^{-+}$resonance has gained wider acceptance. This second resonance was observed by the E852 Collaboration [4] at the slightly higher mass of $1593 \pm 8 \mathrm{MeV}$ and with a width of $168 \pm 20 \mathrm{MeV}$. With the construction of a new experimental Hall D and an energy upgrade proposed for the Thomas Jefferson National Accelerator Facility, the exotic meson spectrum will be probed with an accuracy hitherto unknown; the data gathered should provide compelling evidence, either for or against, the existence and masses of these two $J^{P C}$-exotic states.

Mesons with so-called exotic quantum numbers $J^{P C}=$ $0^{--}, 0^{+-}, 1^{-+}, 2^{+-}$, etc., are traditionally interpreted within a quantum mechanical framework as either hybrids or molecules; that is, $q \bar{q} g$ or $q \bar{q} q \bar{q}$ states, respectively [5]. The conventional quantum mechanical picture of a meson is that it is comprised of a quarkantiquark state $|q \bar{q}\rangle$ and so must have a space-inversion parity of $P=(-1)^{L+1}$, and a charge-conjugation parity of $C=(-1)^{L+S}$, where $L$ is the relative orbital angular momentum and $S$ is the total spin of the system. Therefore, it is impossible to construct a quark-antiquark state that has $J^{P C}=1^{-+}$quantum numbers. Analogously, lattice-QCD simulations of the exotic meson spectrum employ operators that contain explicit excitations of the gluon degrees of freedom. This is done either by joining quark sources by link operators corresponding to trans- verse excitations of the color flux tube [6] or by combining plaquette excitations with quark bilinears [7].

In what may appear to be in contradiction to the aforementioned approaches, one finds that in a fully-covariant quantum field theoretic treatment of meson bound states, one can in fact construct quark bilinear operators which have $J^{P C}$-exotic quantum numbers without the introduction of explicit gluon degrees of freedom. This is a result of having an additional degree of freedom available in quantum field theory arising from the relative-time between the quark and antiquark, leading to an additional factor of $(-1)^{\kappa}$ in the charge-conjugation parity relation $C=(-1)^{L+S+\kappa}$, where $\kappa$ is the relative-time quantum number analogous to $L$. The usual even-time parity operators employed within studies of non-exotic mesons have $\kappa=0,2,4, \ldots$. Operators for which $\kappa$ is an odd integer are referred to as "odd-time parity" operators. Although the existence of two-body bound-state solutions with $J^{P C}$-exotic quantum numbers has been known since the earliest studies of the Bethe-Salpeter equation [8], the details of their role and interpretation within quantum field theory remain unresolved.

It should be emphasized that the use of such odd-timeparity operators, which are devoid of explicit gluon degrees of freedom, to generate exotic meson solutions from the BS equation does not imply that exotic mesons have a non-zero overlap with the state $|q \bar{q}\rangle$. Rather, since such operators have $\kappa \neq 0$, they vanish when the relative time between the quark and antiquark is zero, and hence have no overlap with a $|q \bar{q}\rangle$ state. That is, the states with the fewest number of particles that could appear in a Fock space expansion of an exotic meson would be states such as $|\bar{q} q \bar{q} q\rangle$ and $|\bar{q} q g\rangle$. This is, of course, consistent with the convention picture described above.

In principle, one could also employ similar quark bilinear operators in lattice-QCD simulations. Some examples of suitable operators for the $1^{-+}$-exotic meson would be the lattice versions of $\bar{\psi} \gamma_{i} D_{4} \psi$ and $\bar{\psi} \gamma_{4} D_{i} \psi$ which are constructed using the gauge-covariant derivative $D_{\mu}=\partial_{\mu}+i e A_{\mu}$. In a framework, invariant un- 
der local SU(3)-color transformations, the use of such covariant derivatives inextricably mixes odd-time parity quark bilinear operators with the usual quark-antiquarkgluon hybrid states considered in other models. However, in practice lattice-QCD simulations are typically carried out at quark masses greater than the strange quark mass and hence are uncomfortably close to the non-relativistic limit. Because odd-time-parity operators go to zero in the limit of infinitely heavy quarks, their use in lattice-QCD calculations is not promising as any signal is likely to be swamped by noise [6]. In contrast to lattice gauge theory, this poses no difficulty in the present study, as the Dyson-Schwinger framework has proved to be directly applicable to the limit of small current quark masses. Hence, odd-time-parity operators may provide a useful tool for the study of $J^{P C}$-exotic mesons within this framework.

In the present study of the $1^{-+}$-exotic spectrum, we employ a quantum field theoretic framework based on the Dyson-Schwinger and Bethe-Salpeter (BS) equations [9]. The use of quark bilinear, odd-time parity operators with $J^{P C}$-exotic quantum numbers allows one to obtain solutions of the homogeneous quark-antiquark BS equation that have non-zero overlap with $J^{P C}$-exotic mesons. In this way, we obtain mass predictions for exotic mesons without the explicit introduction of gluon degrees of freedom.

\section{BETHE-SALPETER EQUATION FOR VECTOR AND AXIALVECTOR MESONS}

Our starting point is a calculation of the ground state spectrum of light-quark mesons in which a confining, separable Ansatz for the BS kernel is constructed from phenomenologically efficacious, confined quark propagators [10]. The model was used to calculate the masses for the ground state flavor-octet scalar, psuedoscalar, vector, and $J^{P C}=1^{-+}$axialvector mesons with remarkable success. The authors also explored the possibility of exoticscalar and exotic-pseudoscalar states. For the lightest of these mesons with $J^{P C}=0^{+-}$and $0^{--}$quantum numbers, they obtained masses of 1082 and $1319 \mathrm{MeV}$, respectively. The value for exotic-pseudoscalar meson seems plausible, although there is no experimental consensus regarding the mass of such a state at present. However, the mass of the exotic-scalar meson appears too light. This may be the result of a known weakness of ladder-like Bethe-Salpeter kernels in the study of scalar mesons [11]. That weakness is evident in Ref. 10] through the predicted mass of the $0^{++}$mesons: $749 \mathrm{MeV}$, which is too light to be satisfactorily identified with the $f_{0}$ and $a_{0}$, but too heavy for the putative $\sigma$-meson. On the other hand, the results in Ref. 10 are robust when it comes to predicting masses of pseudoscalar, vector and axialvector mesons, typically giving results within a few percent of the experimental values. This outcome can also be anticipated [12]. If this pattern is maintained in exotic channels, one expects that the mass obtained in Ref. [10] for the exotic-scalar meson is unreliable, while masses obtained for the exotic-pseudoscalar channel, as well as the exotic-vector meson channel considered in this study, would be dependable.

Before proceeding, one should note that exotic-meson solutions of the BSE do not arise as a result of the use of a separable-kernel Ansatz, like the one employed in Ref. [10]. Such solutions have appeared in many different Bethe-Salpeter calculations and are also expected to arise in more sophisticated models that use local, dressedgluon exchange kernels, such as the very successful model considered in Refs. [13].

The quark propagators used herein are based on numerical solutions of model quark Dyson-Schwinger equations, and are algebraic functions fit to a broad range of pion and kaon observables, including the decay constants $f_{\pi}$ and $f_{K}$, charge radii $r_{\pi}$ and $r_{K}, \pi$ - $\pi$ scattering lengths, the pion and kaon electrodynamic form factor $F_{\pi}\left(Q^{2}\right)$ and $F_{K}\left(Q^{2}\right)$, and the anomalous $\gamma \pi \rightarrow \gamma$ and $\gamma \pi \rightarrow \pi \pi$ transition form factors [14. Quark confinement is explicitly included in this model by the fact that the quark propagator $S(p)$ is analytic for all finite values of the quark momentum $p$ on the complex- $p$ plane. From this, it follows that this quark propagator has no Lehmann representation and describes the propagation of a confined quark [9].

The calculation of meson bound states consists of solving the quark-antiquark BS equation in the dressedladder approximation

$$
\begin{aligned}
\Gamma_{\alpha}(p, P)= & -\frac{4}{3} \int \frac{d^{4} q}{(2 \pi)^{4}} g^{2} D_{\mu \nu}(p-q) \gamma_{\mu} S\left(q+\frac{1}{2} P\right) \\
& \times \Gamma_{\alpha}(q, P) S\left(q-\frac{1}{2} P\right) \gamma_{\nu},
\end{aligned}
$$

where $\Gamma_{\alpha}(q, P)$ is the BS amplitude, $S(p)=-i \gamma$. $p \sigma_{V}\left(p^{2}\right)+\sigma_{S}\left(p^{2}\right)$ is the Euclidean-space confined-quark propagator for $u$ and $d$ quarks,

$$
K(p, q)=-\frac{4}{3} g^{2} \gamma_{\mu} D_{\mu \nu}(p-q) \gamma_{\nu},
$$

is the dressed-ladder approximation to the quarkantiquark scattering kernel, and the hermitian Euclidean Dirac matrices $\gamma_{\mu}$ satisfy $\left\{\gamma_{\mu}, \gamma_{\nu}\right\}=2 \delta_{\mu \nu}$. By choosing for the kernel $K(p, q)$, the separable-gluon Ansatz

$$
g^{2} D_{\mu \nu}(p-q)=\delta_{\mu \nu}\left[G\left(p^{2}\right) G\left(q^{2}\right)+p \cdot q F\left(p^{2}\right) F\left(q^{2}\right)\right],
$$

the Dyson-Schwinger equation for the quark propagator can be inverted to give an algebraic relation between the kernel functions $F\left(p^{2}\right)$ and $G\left(p^{2}\right)$ and the Lorentzinvariant functions $\sigma_{V}\left(p^{2}\right)$ and $\sigma_{S}\left(p^{2}\right)$ in the quark propagator $S(p)$. The model kernel is completely determined once the two functions $\sigma_{V}\left(p^{2}\right)$ and $\sigma_{S}\left(p^{2}\right)$ are specified. 
TABLE I: Normalized amplitudes and bound-state masses obtained from Bethe-Salpeter equation for axial, vector, and exoticvector mesons are compared with the observed masses from [1]. All masses are given in units of $\mathrm{MeV}$.

\begin{tabular}{c|c|rrr|c|c}
$J^{P C}$ & $M$ & $\lambda_{1}$ & $\lambda_{2}$ & $\lambda_{3}$ & Identification & Observed Masses \\
\hline $1^{--}$ & 736 & -0.075 & 0.331 & -0.049 & $\rho(770), \omega(782)$ & $770 \pm 1,782 \pm 0$ \\
$1^{+-}$ & 1244 & 0.017 & 0.587 & 0.145 & $h_{1}(1170), b_{1}(1235)$ & $1170 \pm 20,1230 \pm 3$ \\
$1^{++}$ & 1337 & 0.056 & -0.276 & & $a_{1}(1260), f_{1}(1285)$ & $1230 \pm 40,1282 \pm 1$ \\
$1^{-+}$ & 1439 & 0.299 & -0.053 & & $\pi_{1}(1400)$ & $1376 \pm 17$ \\
$1^{-+}$ & 1498 & 0.040 & 0.251 & & $\pi_{1}(1600)$ & $1593 \pm 8$
\end{tabular}

These functions are fit to a range of pion and kaon observables in Ref. [10] and have been successfully applied to study a plethora of other meson reactions [14]. The resulting solutions of the BS equation using the separable kernel of Eq. (3) provide an excellent description of the ground state flavor octet pseudoscalar, vector and axialvector meson spectrum 10.

Restrictions placed on BS amplitudes by Lorentz covariance, space inversion, charge conjugation and the separable Ansatz of Eq. (3), imply that the most general forms of the transverse components $\Gamma_{i}, i=1,2,3$ of the $J^{P C}=1^{--}, 1^{+-}, 1^{++}$and $1^{-+}$amplitudes can be obtained from 10

$$
\Gamma_{i}^{--}(q, P)=q_{i} F\left(q^{2}\right) \lambda_{1}+i \gamma_{i} G\left(q^{2}\right) \lambda_{2}
$$

The masses that result from our calculations for the lightest vector, axialvector and two $1^{-+}$-exotic mesons

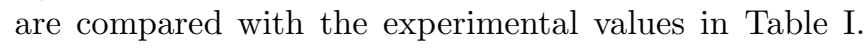
The masses obtained for the non-exotic vector and axialvector mesons are each within $10 \%$ of their accepted values. The results for the $J^{P C}=1^{--}(\rho / \omega)$ and $1^{++}$ $\left(a_{1} / f_{1}\right)$ have been published previously 10 , and recently, calculations of the masses and decays of the $1^{+-} h_{1}(1170)$ and $b_{1}(1235)$ mesons have also appeared [15]. The new results of the present study are the two solutions of the BS equation with $1^{-+}$-exotic quantum numbers. The first has a mass of $1439 \mathrm{MeV}$ and the second has a mass of $1498 \mathrm{MeV}$. These results are truly predictions of the model in that none of the parameters determined in Ref. [10] have been adjusted for the calculations of any of the mesons obtained here.

It is interesting that the mass predictions obtained herein for the exotic-vector meson are somewhat lower than the range $1700-2000 \mathrm{MeV}$ obtained within quantum mechanical models [5] and lattice-QCD simulations [16]. Our results are in the range of mass values of 1300-1700 MeV obtained from bag model calculations [17] (although these values are believed to suffer from parameter uncertainties [18]), and in the large range of

$$
\begin{gathered}
\quad+\gamma_{5} \epsilon_{i j k} \gamma_{j} q_{k} F\left(q^{2}\right) \lambda_{3} \\
\Gamma_{i}^{+-}(q, P)=\quad i \gamma_{5} \gamma_{i} q_{4} F\left(q^{2}\right) \lambda_{1}+i \gamma_{5} q_{i} F\left(q^{2}\right) \lambda_{2} \\
\\
\quad+i \gamma_{5} q_{i} \gamma_{4} F\left(q^{2}\right) \lambda_{3} \\
\Gamma_{i}^{++}(q, P)=i \gamma_{5} \gamma_{i} G\left(q^{2}\right) \lambda_{1}+\epsilon_{i j k} \gamma_{j} q_{k} F\left(q^{2}\right) \lambda_{2}, \\
\Gamma_{i}^{-+}(q, P)=\gamma_{i} q_{4} F\left(q^{2}\right) \lambda_{1}+q_{i} \gamma_{4} F\left(q^{2}\right) \lambda_{2}
\end{gathered}
$$

where the coefficients $\lambda_{A}$ are obtained by solving the BS equation for $P^{2}=-M^{2}$, with $M$ being the mass of the meson bound state under consideration. The resulting coefficients $\lambda_{A}$ for each of the channels considered in Eqs. (4)-(7) are given, along with the corresponding value of $M$, in Table $\mathbb{1}$. The coefficients have been normalized using the formula Eq. (8) discussed below.

values obtained using QCD sum rules 19 for which the lightest mass for exotic- $1^{-+}$mesons varies between 1300 $2100 \mathrm{MeV}$.

Our calculations suggest that there should be two $1^{-+}$. exotic mesons in close proximity to each other. If this is true, then one expects the extraction of their properties from experimental data to be further complicated by these overlapping resonances, each of which is anticipated to have a width of $150-300 \mathrm{MeV}$. However, the separable nature of the model gluon propagator $D_{\mu \nu}(p-q)$ in Eq. (2) that leads to the exotic-vector meson BetheSalpeter amplitude in Eq. (7) only supports two bound states in the $J^{P C}=1^{-+}$channel. As a result, the higher of these two bound states may be artificially lower in the spectrum than one would expect from results from similar calculations based on a non-separable kernel. The existence of higher mass exotic mesons is an issue that clearly needs to be pursued further within more sophisticated models.

One important ramification of including odd-time parity quark-bilinear operators (such as those considered hererin) in a study of exotic mesons is the impact such operators have on the decay modes of these mesons. In particular, it has been argued that the decay of an exotic- 
$1^{-+}$meson into a $\pi$ and $\eta$ final state is suppressed by the Okubo-Zweig-Iuzaki rule 20]. Such a suppression depends critically on the assumption that the structure of the exotic meson is given by a $\bar{q} q g$ operator. On the other hand, if the exotic meson contained a significant mixing of the quark-bilinear operator of the form given in Eq. (7), then such a suppression would not be expected; the exotic meson decay could have a significant branching ratio into the $\pi \eta$ channel. It is interesting to note that nearly all of the decay events observed by experiment so far are, in fact, $\pi_{1}(1400) \rightarrow \eta \pi$. Additional studies are necessary to explore the importance of various operators on exotic-meson decays.

Before concluding, a comment concerning the normalization of the exotic BS amplitudes is in order. Historically, solutions to the homogeneous BS equation with exotic quantum numbers have been thought to be associated with "negative norm states"; that is, the sign of the residue of the odd-time parity pole is opposite to that of a usual meson. This feature of the BS equation has been explored within many model calculations [8], and we observe similar behavior in the present study.

The normalization condition for BS amplitudes can be derived by considering the residue of the bound state pole appearing in the 4-point quark-antiquark scattering amplitude. When the BS kernel is independent of the total meson momentum $P$, as is the case here, the normalization condition for vector (or axialvector) mesons is given by

$$
\begin{gathered}
2 T_{\alpha \beta}(P) P_{\mu}=N_{C} \int \frac{d^{4} k}{\left(2 \pi^{4}\right)} \operatorname{tr}[ \\
\Gamma_{\alpha}(k,-P) \frac{\partial}{\partial P_{\mu}} S\left(k+\frac{1}{2} P\right) \Gamma_{\beta}(k, P) S\left(k-\frac{1}{2} P\right) \\
\left.+\Gamma_{\alpha}(k,-P) S\left(k+\frac{1}{2} P\right) \Gamma_{\beta}(k, P) \frac{\partial}{\partial P_{\mu}} S\left(k-\frac{1}{2} P\right)\right]
\end{gathered}
$$

where $N_{C}=3, T_{\alpha \beta}(P)=\delta_{\alpha \beta}-P_{\alpha} P_{\beta} / P^{2}$ is the spin-one projection operator and "tr" denotes a trace over Dirac indices. There is an analogous expression in Minkowski space.

The result of using this canonical normalization relation, Eq. (8), is that the exotic-vector meson BS amplitude does indeed have a negative normalization. (The remaining states given in Table 1 have a positive normalization.) One possible ramification of a negative BS normalization would be seen in quark-antiquark scattering amplitude, at least in principle. A negative BS normalization is associated with the appearence of the pole in the quark-antiquark scattering amplitude $\mathcal{M}$ with a negative residue. However, this possibility does not immediately cause any problems for two reasons. First, on-shell quark-antiquark scattering amplitudes (even if such objects did exist) do not couple to exotic-vector mesons because the overall factor of $q \cdot P=0$ in Eq. (7) precludes such couplings to on-shell fermions. Second, consequences of quantum mechanical relations such as unitarity and the optical theorem, which could have non-trivial complications with the appearence of negative residue states since they relate off-shell amplitudes to each other, are here, too, avoided. They cause no problems since unitarity relations receive no contribution from single-particle intermediate states [21]. This simple observation is due to the fact that if the one-particle state is stable it must be lighter than any two-particle threshold, and so is not directly produced in two-particle scattering. Conversely, if the one-particle state is unstable then it is never observed asyptotically and it does not play a role in unitarity constraints; no particle flux is carried away by unstable states. Problems that might arise due to exotic mesons having negative BS normalizations would first appear in the $J^{P C}=1^{-+}$channel of two-hadron scattering, such as $\pi b_{1}$ scattering.

The real issue that must be addressed to fully determine the nature of exotic-vector mesons obtained from the BSE is whether or not, in the absence of open decay channels, the resulting exotic-meson state has a positive norm in the Hilbert space. In the present quantum field theoretic framework, this question requires an analytic continuation of our amplitudes into Minkowski space and then a reconstruction of the Hilbert space by means of the techniques and theorems developed in Wightman axiomatic field theory. Such a program is beyond the scope of the present study, although there is progress towards these goals. In particular, a procedure to carry out the first step of explicit analytic continuation from Euclidean to Minkowski space for amplitudes in confined-quark models is currently being successfully employed by one of the authors.

For the moment, a complete understanding of the role of exotic mesons in the BSE and the ramifications of a negative BS normalization remains elusive. Still, it is our belief that any possible difficulties will be overcome in the future since if exotic mesons do exist in nature, then there seems to be no reason a-priori why one could not construct non-local $\bar{q} q$ interpolating fields of the form of the BS amplitude in Eq. (7). Such interpolating fields could then be used to generate driving terms in quark-antiquark scattering equations resulting in the appearence of poles in exotic-meson channels, the observed exotic-meson states. The existence of such poles, in turn, imply the homogeneous BSE, Eq. (1), considered in this study.

\section{CONCLUSION}

In conclusion, we have obtained masses for two $1^{-+}$ exotic mesons from the quark-antiquark Bethe-Salpeter equation by assuming an odd-time-parity structure of the BS amplitude. The masses obtained for the exotic- $1^{-+}$ mesons are 1439 and $1498 \mathrm{MeV}$. The mass of the lighter 
of the two exotics is significantly lighter than results obtained by most other models, but seems to be in good agreement with current experimental values.

It is shown that the odd-time-parity amplitudes associated with these exotic-vector mesons do indeed correspond to bound states whose Bethe-Salpeter amplitudes have negative normalizations as found in earlier studies. Nonetheless, one can argue that this by itself is not enough to disregard solutions of the Bethe-Salpeter equation in these channels, and additional studies employing more realistic models are necessary before determining the meaning of negative BS normalizations as well as any possible pathologies that may arise from such solutions.

It is interesting that the odd-time-parity operators appearing in the structure of exotic mesons may provide additional decay channels for exotic mesons, such as $\pi_{1}(1400) \rightarrow \eta \pi$, that would normally be suppressed according to the OZI rule. This would provide a novel solution to the fact that while some models predict an insignificant branching width for the decay $\pi_{1}(1400) \rightarrow \eta \pi$, experiments find this to be a dominant decay mode.

This work is supported by the National Science Foundation under contract PHY0071361.

[1] Particle Data Group, Eur. Phys. J. C 15, 1 (2000).

[2] D. R. Thompson et al. (E852 Collab.) Phys. Rev. Lett. 79, 1630 (1997).

[3] A. Abele, et al., (Crystal Barrel Collab.) Phys. Lett. B 423, 175 (1998).

[4] G. S. Adams, et al., (E852 Collab.) Phys. Rev. Lett. 81, 5760 (1998).

[5] P. R. Page, Phys. Lett. B 415, 205 (1997); T. Barnes, F. E. Close and E. S. Swanson, Phys. Rev. D 52, 5242 (1995); Yu. S. Kalashnikova and Yu. Yufryakov, Phys. Lett. B 359, 175 (1995); Phys. Atom. Nucl. 60, 307 (1997).

[6] P. Lacock et al., Phys. Rev. D 54, 6997 (1996); P. Lacock et al., Phys. Lett. B 401, 308 (1997).

[7] C. Bernard et al., Phys. Rev. D 56, 7093 (1997).

[8] N. Nakanishi, Phys. Rev. 138B, 1182 (1965); C. H. Llewellyn Smith, Ann. Phys. 53, 521 (1969); M. Ciafoloni, Nuovo Cim. 51 A, 1090 (1967); M. Böhm,
H. Joos and M. Krammer, Nucl. Phys. B51, 397 (1973); A. H. Guth, Ann. Phys. 82, 407 (1974); C. H. Llewellyn Smith, Nuovo Cim. 60A, 348 (1969).

[9] R. Alkofer and L. von Smekal, Phys. Rept. 353, 281 (2001); C. D. Roberts and S. M. Schmidt, Prog. Part. Nucl. Phys. 45, S1 (2000); C. D. Roberts and A. G. Williams, Prog. Part. Nucl. Phys. 33447 (1994); C. D. Roberts, Fiz. Élem. Chastits At. Yadra 30537 (1999).

[10] C. J. Burden, et al., Phys. Rev. C 55, 2649 (1997).

[11] A. Bender, C.D. Roberts and L. v. Smekal, Phys. Lett. B 380, 7 (1996); C.D. Roberts, "Confinement, diquarks and Goldstone's theorem," in Proc. of the 2nd International Conference on Quark Confinement and the Hadron Spectrum, edited by N. Brambilla and G.M. Prosperi (World Scientific, Singapore, 1997) pp. 224-230.

[12] A. Bender, W. Detmold, C.D. Roberts and A.W. Thomas, nucl-th/0202082.

[13] P. Maris and P.C. Tandy, Phys. Rev. C 60, 044214 (1999); P. Maris, "Electromagnetic, weak, and strong interactions of light mesons," arXiv:nucl-th/0112022.

[14] C. D. Roberts, Nucl. Phys. A 605, 475 (1996); M. R. Frank et al., Phys. Lett. B 359, 17 (1995); R. Alkofer and C. D. Roberts, Phys. Lett. B 369, 101 (1996).

[15] J. C. R. Bloch, Yu. L. Kalinovsky, C. D. Roberts, S. M. Schmidt, Phys. Rev. D 60 (Rapid Comm.) 111502 (1999).

[16] C. McNeil, Latest results from lattice QCD for exotic hybrid mesons, talk presented at Workshop on Hadron Spectroscopy, INFN, hep-lat/9904013, and references therein.

[17] T. Barnes and F. E. Close, Phys. Lett. B 116, 365 (1982); T. Barnes, F. E. Close and F. de Viron, Nucl. Phys. B 224, 241 (1983); M. Chanowitz and S. Sharpe, Nucl. Phys. B 222, 211 (1983).

[18] A. Donnachie and Yu. S. Kalashnikova, Phys. Rev. D 60 114011 (1999).

[19] I. I. Balitsky, D. I. Dyakonov and A. V. Yung, Z. Phys. C 33, 265 (1986); J. I. Lattore, P. Pascual and S. Narison, Z. Phys. C 34, 347 (1987); J. Govaerts et al., Phys. Lett. B 128, 262 (1983); 136, 445E (1983); Nucl. Phys. B 248, 1 (1984).

[20] F. Iddir, A. Le Yaouanc, L. Oliver, O. Pene and J. C. Raynal, Phys. Lett. B 207, 325 (1988).

[21] M. A. Pichowsky, A. Szczepaniak and J. T. Londergan, Phys. Rev. D 64, 036009 (2001). 